Eigenvalue perturbation bounds for Hermitian block tridiagonal matrices

Nakatsukasa, Yuji

2011

MIMS EPrint: 2011.81

Manchester Institute for Mathematical Sciences

School of Mathematics

The University of Manchester

\footnotetext{
Reports available from: http://eprints.maths.manchester.ac.uk/

And by contacting: The MIMS Secretary

School of Mathematics

The University of Manchester

Manchester, M13 9PL, UK
} 


\title{
Eigenvalue perturbation bounds for Hermitian block tridiagonal matrices
}

\author{
Yuji Nakatsukasa ${ }^{\mathrm{a}, 1, *}$ \\ ${ }^{a}$ School of Mathematics, The University of Manchester, Manchester, M13 9PL, UK
}

\begin{abstract}
We derive new perturbation bounds for eigenvalues of Hermitian matrices with block tridiagonal structure. The main message of this paper is that an eigenvalue is insensitive to blockwise perturbation, if it is well-separated from the spectrum of the diagonal blocks nearby the perturbed blocks. Our bound is particularly effective when the matrix is block-diagonally dominant and graded. Our approach is to obtain eigenvalue bounds via bounding eigenvector components, which is based on the observation that an eigenvalue is insensitive to componentwise perturbation if the corresponding eigenvector components are small. We use the same idea to explain two well-known phenomena, one concerning aggressive early deflation used in the symmetric tridiagonal QR algorithm and the other concerning the extremal eigenvalues of Wilkinson matrices.
\end{abstract}

Keywords: eigenvalue perturbation, Hermitian matrix, block tridiagonal, Wilkinson's matrix, aggressive early deflation

2000 MSC: $15 \mathrm{~A} 22 ; 15 \mathrm{~A} 42 ; 65 \mathrm{~F} 15$

\section{Introduction}

Eigenvalue perturbation theory for Hermitian matrices is a well-studied subject with many known results, see for example [21, Ch.4] [8, Ch.8], [7, Ch.4]. Among them, Weyl's theorem is perhaps the simplest and most well-known, which states that the eigenvalues of the Hermitian matrices $A$ and $A+E$ differ at most by $\|E\|_{2}$. In fact, when the perturbation $E$ is allowed to be an arbitrary Hermitian matrix, Weyl's theorem gives the smallest possible bound that is attainable.

Hermitian matrices that arise in practice frequently have special sparse structures, important examples of which being banded and block tridiagonal structures. For such structured matrices, perturbation of some eigenvalues is often much smaller than any known bound guarantees. The goal of this paper is to treat block tridiagonal Hermitian matrices and derive eigenvalue perturbation bounds that can be much sharper than known general bounds, such as Weyl's theorem.

The key observation of this paper, to be made in section 2 , is that an eigenvalue is insensitive to componentwise perturbations if the corresponding eigenvector components are small. Our approach is to obtain bounds for eigenvector components, from which we obtain eigenvalue bounds.

\footnotetext{
*Corresponding author

Email address: yuji.nakatsukasa@manchester .ac.uk (Yuji Nakatsukasa)

${ }^{1}$ Phone: 44-0161-306-3640
} 
In this framework we first give new eigenvalue perturbation bounds for the simplest, 2-by-2 block case. In particular, we identify a situation in which the perturbation bound of an eigenvalue scales cubically with the norm of the perturbation.

We then discuss the general block tridiagonal case, in which we show that an eigenvalue is insensitive to blockwise perturbation, if it is well-separated from the spectrum of the diagonal blocks nearby the perturbed blocks.

Finally, to demonstrate the effectiveness of our approach, we show that our framework successfully explains the following two well-known phenomena: (i) Aggressive early deflation applied to the symmetric tridiagonal QR algorithm deflates many eigenvalues even when no off-diagonal element is negligibly small. (ii) Wilkinson matrices have many pairs of nearly equal eigenvalues.

A number of related studies exist in the literature, especially in the tridiagonal case for which explicit formulas exist for the eigenvector components using the determinants of submatrices [19, Sec. 7.9]. Cuppen [5] gives an explanation for the exponential decay in eigenvector components of tridiagonal matrices, which often lets the divide-and-conquer algorithm run much faster than its estimated cost suggests. The derivation of our eigenvector bounds are much in the same vein as Cuppen's argument. A difference here is that we use the bounds to show the insensitivity of eigenvalues. In [18] Parlett investigates the localization behavior of eigenvectors (or an invariant subspace) corresponding to a cluster of $m$ eigenvalues, and notes that accurate eigenvalues and nearly orthogonal eigenvectors can be computed from appropriately chosen $m$ submatrices, which allow overlaps within one another. One implication of this is that setting certain subdiagonals to zero has negligible influence on some of the eigenvalues. A similar claim is made by our Theorem 4.2, which holds for block tridiagonal matrices, whether or not the eigenvalue belongs to a cluster. In addition, in a recent paper [20] Parlett considers symmetric banded matrices and links the disjointness of an eigenvalue from Gerschgorin disks with bounds of off-diagonal parts of $L$ and $U$ in the $L D U$ decomposition, from which exponential decay in eigenvector components can be deduced. A similar message is conveyed by our Lemma 4.1, but unlike [20] we give direct bounds for the eigenvector components, and we use them to obtain eigenvalue bounds. Moreover, Parlett and Vömel [17] study detecting such eigenvector decay behavior to devise a process to efficiently compute some of the eigenvalues of a symmetric tridiagonal matrix. Our results in this paper may be used to foster such developments. Finally, [10] considers general Hermitian matrices and shows for any eigenpair $\left(\lambda_{i}, x\right)$ of $A$ that the interval $\left[\lambda_{i}-\|E x\|_{2}, \lambda_{i}+\|E x\|\right]$ contains an eigenvalue of $A+E$, and gives a condition under which the eigenvalue is the $i$ th eigenvalue. Our approach here is roughly to give explicit bounds for $\|E x\|_{2}$ for the block tridiagonal case, while maintaining the one-to-one correspondence between the $i$ th eigenvalue of $A$ and that of $A+E$.

The rest of this paper is organized as follows. In section 2 we outline our basic idea of deriving eigenvalue perturbation bounds via bounding eigenvector components. Section 3 treats the 2-by-2 block case and presents a new bound. Section 4 discusses the block tridiagonal case. In section 5 we investigate the two case studies.

Notations: $\lambda_{i}(X)$ denotes the $i$ th smallest eigenvalue of a Hermitian matrix $X$. For simplicity we use $\lambda_{i}, \widehat{\lambda}_{i}$ and $\lambda_{i}(t)$ to denote the $i$ th smallest eigenvalue of $A, A+E$ and $A+t E$ for $t \in[0,1]$ respectively. $\lambda(A)$ denotes $A$ 's spectrum, the set of eigenvalues. We use only the matrix spectral norm $\|\cdot\|_{2}$. 


\section{Basic approach}

We first recall the partial derivative of a simple eigenvalue [21].

Lemma 2.1. Let $A$ and $E$ be n-by-n Hermitian matrices. Denote by $\lambda_{i}(t)$ the ith eigenvalue of $A+t E$, and define the vector-valued function $x(t)$ such that $(A+t E) x(t)=\lambda_{i}(t) x(t)$ where $\|x(t)\|_{2}=1$ for some $t \in[0,1]$. If $\lambda_{i}(t)$ is simple, then

$$
\frac{\partial \lambda_{i}(t)}{\partial t}=x(t)^{H} E x(t)
$$

Our main observation here is that if $x(t)$ has small components in the positions corresponding to the dominant elements of $E$, then $\frac{\partial \lambda_{i}(t)}{\partial t}$ is small. For example, suppose that $E$ is nonzero only in the $(j, j)$ th element. Then we have $\left|\frac{\partial \lambda_{i}(t)}{\partial t}\right| \leq\|E\|_{2}\left|x_{j}(t)\right|^{2}$, where $x_{j}(t)$ is $j$ th element of $x(t)$. Hence if we know a bound for $\left|x_{j}(t)\right|$ for all $t \in[0,1]$, then we can integrate (1) over $0 \leq t \leq 1$ to obtain a bound for $\left|\lambda_{i}-\widehat{\lambda}_{i}\right|=\left|\lambda_{i}(0)-\lambda_{i}(1)\right|$. In the sequel we shall describe in detail how this observation can be exploited to derive eigenvalue perturbation bounds.

It is important to note that Lemma 2.1 assumes that $\lambda_{i}$ is a simple eigenvalue of $A$. Special treatment is needed to get the derivative of multiple eigenvalues. This is described in the appendix, in which we show that everything we discuss below carries over even in the presence of multiple eigenvalues. In particular, when $\lambda_{i}(t)$ is multiple (1) still holds for a certain choice of eigenvector $x(t)$ of $\lambda_{i}(t)$. We defer the treatment of multiple eigenvalues to the appendix, because it only causes complications to the analysis that are not fundamental to the eigenvalue behavior. Hence for simplicity until Appendix A we assume that $\lambda_{i}(t)$ is simple for all $t$, so that the normalized eigenvector is unique up to a factor $e^{\mathrm{i} \theta}$.

\section{2-by-2 block case}

In this section we consider the 2-by-2 case. Specifically, we study the difference between eigenvalues of the $n$-by- $n$ Hermitian matrices $A$ and $A+E$, where

$$
A=\left[\begin{array}{ll}
A_{11} & A_{21}^{H} \\
A_{21} & A_{22}
\end{array}\right] \text { and } E=\left[\begin{array}{ll}
E_{11} & E_{21}^{H} \\
E_{21} & E_{22}
\end{array}\right]
$$

in which $A_{22}$ and $E_{22}$ are both $k$-by- $k$.

Since $\lambda_{i}(0)=\lambda_{i}$ and $\lambda_{i}(1)=\widehat{\lambda}_{i}$, from (1) it follows that

$$
\begin{aligned}
\left|\lambda_{i}-\widehat{\lambda}_{i}\right| & =\left|\int_{0}^{1} x(t)^{H} E x(t) d t\right| \\
& \leq\left|\int_{0}^{1} x_{1}(t)^{H} E_{11} x_{1}(t) d t\right|+2\left|\int_{0}^{1} x_{2}(t)^{H} E_{21} x_{1}(t) d t\right|+\left|\int_{0}^{1} x_{2}(t)^{H} E_{22} x_{2}(t) d t\right|,
\end{aligned}
$$

where we block-partitioned $x(t)=\left[\begin{array}{l}x_{1}(t) \\ x_{2}(t)\end{array}\right]$ so that $x_{1}(t)$ and $A_{11}$ have the same number of rows. The key observation here is that the latter two terms in (4) are small if $\left\|x_{2}(t)\right\|_{2}$ is small for all $t \in[0,1]$. We obtain an upper bound for $\left\|x_{2}(t)\right\|_{2}$ by the next lemma. 
Lemma 3.1. Suppose that $\lambda_{i} \notin \lambda\left(A_{22}\right)$ is the ith smallest eigenvalue of $A$ as defined in (2). Let $A x=\lambda_{i} x$ such that $\|x\|_{2}=1$. Then, partitioning $x=\left[\begin{array}{l}x_{1} \\ x_{2}\end{array}\right]$ as above we have

$$
\left\|x_{2}\right\|_{2} \leq \frac{\left\|A_{21}\right\|_{2}}{\min \left|\lambda_{i}-\lambda\left(A_{22}\right)\right|} \text {. }
$$

Proof. The bottom $k$ rows of $A x=\lambda_{i} x$ is

$$
A_{21} x_{1}+A_{22} x_{2}=\lambda_{i} x_{2}
$$

so we have

$$
x_{2}=\left(\lambda_{i} I-A_{22}\right)^{-1} A_{21} x_{1} .
$$

Taking norms we get

$$
\left\|x_{2}\right\|_{2} \leq\left\|\left(\lambda_{i} I-A_{22}\right)^{-1}\right\|_{2}\left\|A_{21}\right\|_{2}\left\|x_{1}\right\|_{2} \leq \frac{\left\|A_{21}\right\|_{2}}{\min \left|\lambda_{i}-\lambda\left(A_{22}\right)\right|},
$$

where we used $\left\|x_{1}\right\|_{2} \leq\|x\|_{2}=1$ to get the last inequality.

We note that Lemma 3.1 is just a special case of the Davis-Kahan generalized $\sin \theta$ theorem [6, Thm. 6.1], in which the two subspaces have dimensions 1 and $n-k$. Specifically, (5) bounds the sin of the canonical angle [8, p. 603] between an eigenvector $x$ and the first $n-k$ columns of the identity matrix $I$.

Clearly, if $\lambda_{i} \notin \lambda\left(A_{11}\right)$ then (5) holds with $x_{2}$ replaced with $x_{1}$ and $A_{22}$ replaced with $A_{11}$. This applies to the entire section, but for definiteness we only present results assuming $\lambda_{i} \notin \lambda\left(A_{22}\right)$.

We note that (5) is valid for any $\lambda_{i}$ and its normalized eigenvector $x$, whether or not $\lambda_{i}$ is a multiple eigenvalue. It follows that in the multiple case, all the vectors that are in the corresponding eigenspace satisfy (5).

We now derive refined eigenvalue perturbation bounds by combining Lemmas 2.1 and 3.1. As before, let $\left(\lambda_{i}(t), x(t)\right)$ be the $i$ th smallest eigenpair such that $(A+t E) x(t)=\lambda_{i}(t) x(t)$ with $\|x(t)\|_{2}=1$. When $\min \left|\lambda_{i}-\lambda\left(A_{22}\right)\right|>2\|E\|_{2}$, using (5) we get an upper bound for $\left\|x_{2}(t)\right\|_{2}$ for all $t \in[0,1]:$

$$
\begin{aligned}
\left\|x_{2}(t)\right\|_{2} & \leq \frac{\left\|A_{21}+t E_{21}\right\|_{2}}{\min \left|\lambda_{i}(t)-\lambda\left(A_{22}+t E_{22}\right)\right|} \\
& \leq \frac{\left\|A_{21}\right\|_{2}+t\left\|E_{21}\right\|_{2}}{\min \left|\lambda_{i}(0)-\lambda\left(A_{22}\right)\right|-2 t\|E\|_{2}} \quad(\because \text { Weyl's theorem }) \\
& \leq \frac{\left\|A_{21}\right\|_{2}+\left\|E_{21}\right\|_{2}}{\min \left|\lambda_{i}-\lambda\left(A_{22}\right)\right|-2\|E\|_{2}} .
\end{aligned}
$$

We now present a perturbation bound for $\lambda_{i}$. 
Theorem 3.2. Let $\lambda_{i}$ and $\widehat{\lambda}_{i}$ be the ith eigenvalue of $A$ and $A+E$ as in (2) respectively, and define $\tau_{i}=\frac{\left\|A_{21}\right\|_{2}+\left\|E_{21}\right\|_{2}}{\min \left|\lambda_{i}-\lambda\left(A_{22}\right)\right|-2\|E\|_{2}}$. Then for each $i$, if $\tau_{i}>0$ then

$$
\left|\lambda_{i}-\widehat{\lambda}_{i}\right| \leq\left\|E_{11}\right\|_{2}+2\left\|E_{21}\right\|_{2} \tau_{i}+\left\|E_{22}\right\|_{2} \tau_{i}^{2}
$$

Proof. Substituting (6) into (4) we get

$$
\begin{aligned}
\left|\lambda_{i}-\widehat{\lambda}_{i}\right| & \leq\left|\int_{0}^{1}\left\|E_{11}\right\|_{2}\left\|x_{1}(t)\right\|_{2}^{2} d t\right|+2\left|\int_{0}^{1}\left\|E_{21}\right\|_{2}\left\|x_{1}(t)\right\|_{2}\left\|x_{2}(t)\right\|_{2} d t\right|+\left|\int_{0}^{1}\left\|E_{22}\right\|_{2}\left\|x_{2}(t)\right\|_{2}^{2} d t\right| \\
& \leq\left\|E_{11}\right\|_{2}+2\left\|E_{21}\right\|_{2} \tau_{i}+\left\|E_{22}\right\|_{2} \tau_{i}^{2}
\end{aligned}
$$

which is (7).

Remark 1. We make three points on Theorem 3.2.

- $\tau_{i}<1$ is a necessary condition for (7) to be tighter than the Weyl bound $\|E\|_{2}$. If $\left\|E_{11}\right\|_{2} \ll$ $\|E\|_{2}$ and $\lambda_{i}$ is far from the spectrum of $A_{22}$ so that $\tau_{i} \ll 1$, then (7) is much smaller than $\|E\|_{2}$.

- When $A_{21}, E_{11}, E_{22}$ are all zero (i.e., when a block-diagonal matrix undergoes an off-diagonal perturbation), (7) becomes

$$
\left|\lambda_{i}-\widehat{\lambda}_{i}\right| \leq \frac{2\left\|E_{21}\right\|_{2}^{2}}{\min \left|\lambda_{i}-\lambda\left(A_{22}\right)\right|-2\left\|E_{21}\right\|_{2}},
$$

which shows the perturbation must be $O\left(\left\|E_{21}\right\|^{2}\right)$ if $\lambda_{i}$ is not an eigenvalue of $A_{22}$.

We note that much work has been done for such structured perturbation. For example, under the same assumption of off-diagonal perturbation, $[15,14]$ prove the quadratic residual bounds

$$
\begin{aligned}
\left|\lambda_{i}-\widehat{\lambda}_{i}\right| & \leq \frac{\left\|E_{21}\right\|_{2}^{2}}{\min \left|\lambda_{i}(A)-\lambda\left(A_{22}\right)\right|} \\
& \leq \frac{2\left\|E_{21}\right\|_{2}^{2}}{\min \left|\lambda_{i}(A)-\lambda\left(A_{22}\right)\right|+\sqrt{\min \left|\lambda_{i}(A)-\lambda\left(A_{22}\right)\right|^{2}+4\left\|E_{21}\right\|_{2}^{2}}} .
\end{aligned}
$$

Our bound (7) (or (8)) is not as tight as the bounds in (9). However, (7) has the advantage that it is applicable for a general perturbation, not necessarily off-diagonal.

- (7) also reveals that if $E=\left[\begin{array}{cc}0 & 0 \\ 0 & E_{22}\end{array}\right]$ and $A_{21}$ is small, then $\lambda_{i}$ is particularly insensitive to the perturbation $E_{22}$ : the bound (7) becomes proportional to $\left\|E_{22}\right\|_{2}\left\|A_{21}\right\|_{2}^{2}$.

For example, consider the $n$-by- $n$ matrices $\left[\begin{array}{cc}A_{11} & v \\ v^{H} & \varepsilon\end{array}\right]$ and $\left[\begin{array}{cc}A_{11} & v \\ v^{H} & 0\end{array}\right]$ where $A_{11}$ is nonsingular. These matrices have one pair of eigenvalues that matches up to $\varepsilon$, and $n-1$ pairs that match up to $O\left(\varepsilon\|v\|_{2}^{2}\right)$. Note that when $\|v\|_{2}=O(\varepsilon), \varepsilon\|v\|_{2}^{2}$ scales cubically with $\varepsilon$. 


\section{Block Tridiagonal case}

Here we consider the block tridiagonal case and apply the idea we used above to obtain a refined eigenvalue perturbation bound. Let $A$ and $E$ be Hermitian block tridiagonal matrices defined by

$$
A=\left[\begin{array}{cccc}
A_{1} & B_{1}^{H} & & \\
B_{1} & \ddots & \ddots & \\
& \ddots & \ddots & B_{n-1}^{H} \\
& & B_{n-1} & A_{n}
\end{array}\right] \text { and } E=\left[\begin{array}{cccccc}
\ddots & \ddots & & & \\
\ddots & 0 & 0 & & \\
& 0 & \Delta A_{s} & \Delta B_{s}^{H} & \\
& & \Delta B_{s} & 0 & 0 \\
& & & 0 & \ddots
\end{array}\right],
$$

where $A_{j}, \in \mathbb{C}^{n_{j} \times n_{j}}$ and $B_{j} \in \mathbb{C}^{n_{j+1} \times n_{j}}$. The size of $\Delta A_{s}$ and $\Delta B_{s}$ match those of $A_{s}$ and $B_{s}$. Here we consider perturbation in a single block, so $E$ is zero except for the sth blocks $\Delta A_{s}$ and $\Delta B_{s}$. When more than one block is perturbed we can apply the below argument repeatedly.

We obtain an upper bound for $\left|\lambda_{i}-\widehat{\lambda}_{i}\right|$ by bounding the magnitude of the eigenvector components corresponding to the $s$ th and $(s+1)$ th blocks. As before we let $\left(\lambda_{i}(t), x(t)\right)$ be the $i$ th eigenpair such that $(A+t E) x(t)=\lambda_{i}(t) x(t)$ for $t \in[0,1]$. To prove a useful upper bound for the blocks of the eigenvector $x(t)$ corresponding to $\lambda_{i}(t)$ for all $t \in[0,1]$, we make the following Assumption 1 . Here we say " $a$ belongs to the $j$ th block of $A$ " if

$$
a \in\left[\lambda_{\min }\left(A_{j}\right)-\eta_{j}, \lambda_{\max }\left(A_{j}\right)+\eta_{j}\right] \quad \text { where } \quad \eta_{j}=\left\|B_{j}\right\|_{2}+\left\|B_{j-1}\right\|_{2}+\|E\|_{2},
$$

in which for convenience we define $B_{0}=0, B_{n}=0$. Note that $a$ can belong to more than one block.

Assumption 1. There exists an integer $\ell>0$ such that $\lambda_{i}$ does not belong to the first $s+\ell$ blocks of $A$.

Roughly, the assumption demands that $\lambda_{i}$ is far away from the eigenvalues of $A_{1}, \ldots, A_{s+\ell}$, and that the norms of $E$ and $B_{1}, \ldots, B_{s+\ell}$ are not too large. A typical case where the assumption holds is when $A_{1}, A_{2}, \ldots, A_{n}$ have a graded structure, so that the eigenvalues of $A_{i}$ are smaller (or larger) than those of $A_{j}$ for all $(i, j)$ with $i<j$. For example, consider the tridiagonal matrix

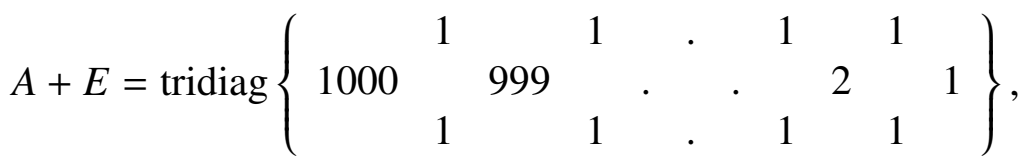

where $E$ is zero except for the first off-diagonals, which are 1 . We set all the block sizes to one, and see that the interval $\left[\lambda_{\min }\left(A_{j}\right)-\eta_{j}, \lambda_{\max }\left(A_{j}\right)+\eta_{j}\right]$ as in (11) for the $j$ th block is $[998,1002]$ for $j=1$ and $[(1001-j)-3,(1001-j)+3]$ for $2 \leq j \leq 999$. Hence for any eigenvalue $\lambda_{i} \leq 100$, $\lambda_{i}$ does not belong to the first 897 blocks, so Assumption 1 is valid with $\ell=896$. More generally, for any $\lambda_{i} \in[k, k+1]$ for any integer $k<997$, Assumption 1 holds with $\ell=997-k$.

We also note that the below argument holds exactly the same for the case where $\lambda_{i}$ does not belong to the last $n-s-\ell$ blocks, but for definiteness we proceed under Assumption 1.

We now derive an upper bound for the eigenvector components. 
Lemma 4.1. Let $A$ and $E$ be Hermitian block tridiagonal matrices as in (10). Suppose that A's ith eigenvalue $\lambda_{i}$ satisfies Assumption 1, so that defining gap $_{j}=\min \left|\lambda_{i}-\lambda\left(A_{j}\right)\right|$, we have gap ${ }_{j}>$ $\|E\|_{2}+\left\|B_{j}\right\|_{2}+\left\|B_{j-1}\right\|_{2}$ for $j=1, \ldots, s+\ell$. Let $(A+t E) x(t)=\lambda_{i}(t) x(t)$ and $\|x(t)\|_{2}=1$ for $t \in[0,1]$, and block-partition $x(t)^{H}=\left[x_{1}(t)^{H} x_{2}(t)^{H} \ldots x_{n}(t)^{H}\right]^{H}$ such that $x_{j}(t)$ and $A_{j}$ have the same numbers of rows. Let $^{2}$

$$
\delta_{0}=\frac{\left\|B_{s}\right\|_{2}+\left\|\Delta B_{s}\right\|_{2}}{g a p_{s}-\|E\|_{2}-\left\|\Delta A_{S}\right\|_{2}-\left\|B_{s-1}\right\|_{2}}, \quad \delta_{1}=\frac{\left\|B_{s+1}\right\|_{2}}{g a p_{s+1}-\|E\|_{2}-\left\|B_{s}\right\|_{2}-\left\|\Delta B_{s}\right\|_{2}},
$$

and

$$
\delta_{j}=\frac{\left\|B_{s+j}\right\|_{2}}{\operatorname{gap}_{s+j}-\|E\|_{2}-\left\|B_{s+j-1}\right\|_{2}} \quad \text { for } \quad j=2, \ldots, \ell,
$$

and suppose that the denominators in (13) and (13) are all positive. Then, for all $t \in[0,1]$ we have

$$
\begin{aligned}
\left\|x_{s}(t)\right\|_{2} & \leq \prod_{j=0}^{\ell} \delta_{j}, \\
\left\|x_{s+1}(t)\right\|_{2} & \leq \prod_{j=1}^{\ell} \delta_{j} .
\end{aligned}
$$

Proof. We first show that $\left\|x_{1}(t)\right\|_{2} \leq\left\|x_{2}(t)\right\|_{2} \leq \cdots \leq\left\|x_{s+\ell}(t)\right\|_{2}$. The first block of $(A+t E) x(t)=$ $\lambda_{i}(t) x(t)$ is

$$
A_{1} x_{1}(t)+B_{1}^{H} x_{2}(t)=\lambda_{i}(t) x_{1}(t)
$$

so we have

$$
x_{1}(t)=\left(\lambda_{i}(t) I-A_{1}\right)^{-1} B_{1}^{H} x_{2}(t) .
$$

Now since by Weyl's theorem we have $\lambda_{i}(t) \in\left[\lambda_{i}-\|E\|_{2}, \lambda_{i}+\|E\|_{2}\right]$ for all $t \in[0,1]$, it follows that $\left\|\left(\lambda_{i}(t) I-A_{1}\right)^{-1}\right\|_{2} \leq 1 /\left(\right.$ gap $\left._{1}-\|E\|_{2}\right)$. Therefore, $\left\|x_{1}(t)\right\|_{2} /\left\|x_{2}(t)\right\|_{2}$ can be bounded by

$$
\frac{\left\|x_{1}(t)\right\|_{2}}{\left\|x_{2}(t)\right\|_{2}} \leq \frac{\left\|B_{1}\right\|_{2}}{g a p_{1}-\|E\|_{2}} \leq 1
$$

where the last inequality follows from Assumption 1.

Next, the second block of $(A+t E) x(t)=\lambda_{i}(t) x(t)$ is

$$
B_{1} x_{1}(t)+A_{2} x_{2}(t)+B_{2}^{H} x_{3}(t)=\lambda_{i}(t) x_{2}(t)
$$

so we have

$$
x_{2}(t)=\left(\lambda_{i}(t) I-A_{2}\right)^{-1}\left(B_{1} x_{1}(t)+B_{2}^{H} x_{3}(t)\right) .
$$

\footnotetext{
${ }^{2} \delta_{j}$ and $g a p_{s}$ depend also on $i$, but we omit the subscript for simplicity.
} 
Using $\left\|\left(\lambda_{i}(t) I-A_{2}\right)^{-1}\right\|_{2} \leq 1 /\left(\operatorname{gap}_{2}-\|E\|_{2}\right)$ we get

$$
\begin{aligned}
\left\|x_{2}(t)\right\|_{2} & \leq \frac{\left\|B_{1}\right\|_{2}\left\|x_{1}(t)\right\|_{2}+\left\|B_{2}\right\|_{2}\left\|x_{3}(t)\right\|_{2}}{g a p_{2}-\|E\|_{2}} \\
& \leq \frac{\left\|B_{1}\right\|_{2}\left\|x_{2}(t)\right\|_{2}+\left\|B_{2}\right\|_{2}\left\|x_{3}(t)\right\|_{2}}{g a p_{2}-\|E\|_{2}}, \quad\left(\because\left\|x_{1}(t)\right\|_{2} \leq\left\|x_{2}(t)\right\|_{2}\right)
\end{aligned}
$$

and so

$$
\frac{\left\|x_{2}(t)\right\|_{2}}{\left\|x_{3}(t)\right\|_{2}} \leq \frac{\left\|B_{2}\right\|_{2}}{\text { gap }_{2}-\|E\|_{2}-\left\|B_{1}\right\|_{2}} .
$$

By Assumption 1 this is no larger than 1 , so $\left\|x_{2}(t)\right\|_{2} \leq\left\|x_{3}(t)\right\|_{2}$.

By the same argument we can prove $\left\|x_{1}(t)\right\|_{2} \leq\left\|x_{2}(t)\right\|_{2} \leq \cdots \leq\left\|x_{s+\ell}(t)\right\|_{2}$ for all $t \in[0,1]$.

Next consider the sth block of $(A+t E) x(t)=\lambda_{i}(t) x(t)$, which is

$$
B_{s-1} x_{s-1}(t)+\left(A_{s}+t \Delta A_{s}\right) x_{s}(t)+\left(B_{s}+t \Delta B_{s}\right)^{H} x_{s+1}(t)=\lambda_{i}(t) x_{s}(t)
$$

so we have

$$
x_{s}(t)=\left(\lambda_{i}(t) I-A_{s}-t \Delta A_{s}\right)^{-1}\left(B_{s-1} x_{s-1}(t)+\left(B_{s}+t \Delta B_{s}\right)^{H} x_{s+1}(t)\right) .
$$

Using $\left\|\left(\lambda_{i}(t) I-A_{s}-t \Delta A_{s}\right)^{-1}\right\|_{2} \leq 1 /\left(\operatorname{gap}_{s}-\|E\|_{2}-\left\|\Delta A_{S}\right\|_{2}\right)$ and $\left\|x_{s-1}(t)\right\|_{2} \leq\left\|x_{s}(t)\right\|_{2}$ we get

$$
\left\|x_{s}(t)\right\|_{2} \leq \frac{\left\|B_{s-1}\right\|_{2}\left\|x_{s}(t)\right\|_{2}+\left\|B_{s}+t \Delta B_{s}\right\|_{2}\left\|x_{s+1}(t)\right\|_{2}}{\text { gap }_{s}-\|E\|_{2}-\left\|\Delta A_{s}\right\|_{2}} .
$$

Hence we get $\frac{\left\|x_{s}(t)\right\|_{2}}{\left\|x_{s+1}(t)\right\|_{2}} \leq \frac{\left\|B_{s}\right\|_{2}+\left\|\Delta B_{s}\right\|_{2}}{\text { gap }_{s}-\|E\|_{2}-\left\|\Delta A_{S}\right\|_{2}-\left\|B_{s-1}\right\|_{2}}=\delta_{0}$ for all $t \in[0,1]$.

The $(s+1)$ th block of $(A+t E) x(t)=\lambda_{i}(t) x(t)$ is

$$
\left(B_{s}+t \Delta B_{s}\right) x_{s}(t)+A_{s+1} x_{s+1}(t)+B_{s+1}^{H} x_{s+2}(t)=\lambda_{i}(t) x_{s+1}(t),
$$

so we get

$$
x_{s+1}(t)=\left(\lambda_{i}(t) I-A_{s+1}\right)^{-1}\left(\left(B_{s}+t \Delta B_{s}\right) x_{s}(t)+B_{s+1}^{H} x_{s+2}(t)\right),
$$

and hence $\frac{\left\|x_{s+1}(t)\right\|_{2}}{\left\|x_{s+2}(t)\right\|_{2}} \leq \frac{\left\|B_{s+1}\right\|_{2}}{g a p_{s+1}-\|E\|_{2}-\left\|B_{s}\right\|_{2}-\left\|\Delta B_{s}\right\|_{2}}=\delta_{1}$. Similarly we can prove that

$$
\frac{\left\|x_{s+j}(t)\right\|_{2}}{\left\|x_{s+j+1}(t)\right\|_{2}} \leq \delta_{j} \quad \text { for } \quad j=1, \ldots, \ell .
$$

Together with $\left\|x_{s+\ell+1}\right\|_{2} \leq\|x\|_{2}=1$ it follows that for all $t \in[0,1]$,

$$
\begin{aligned}
\left\|x_{s}(t)\right\|_{2} & \leq \prod_{j=0}^{\ell} \delta_{j}\left\|x_{s+\ell+1}(t)\right\|_{2} \leq \prod_{j=0}^{\ell} \delta_{j}, \\
\left\|x_{s+1}(t)\right\|_{2} & \leq \prod_{j=1}^{\ell} \delta_{j}\left\|x_{s+\ell+1}(t)\right\|_{2} \leq \prod_{j=1}^{\ell} \delta_{j} .
\end{aligned}
$$


We are now ready to present a perturbation bound for $\lambda_{i}$.

Theorem 4.2. Let $\lambda_{i}$ and $\widehat{\lambda}_{i}$ be the ith eigenvalue of $A$ and $A+E$ as in (10) respectively, and let $\delta_{i}$ be as in (13). Suppose that $\lambda_{i}$ satisfies Assumption 1. Then

$$
\left|\lambda_{i}-\widehat{\lambda}_{i}\right| \leq\left\|\Delta A_{s}\right\|_{2}\left(\prod_{j=0}^{\ell} \delta_{j}\right)^{2}+2\left\|\Delta B_{s}\right\|_{2} \delta_{0}\left(\prod_{j=1}^{\ell} \delta_{j}\right)^{2} .
$$

Proof. Using (3) we have

$$
\begin{aligned}
\left|\lambda_{i}-\widehat{\lambda}_{i}\right| & =\left|\int_{0}^{1} x(t)^{H} \operatorname{Ex}(t) d t\right| \\
& \leq\left|\int_{0}^{1} x_{s}(t)^{H} \Delta A_{s} x_{s}(t) d t\right|+2\left|\int_{0}^{1} x_{s+1}(t)^{H} \Delta B_{s} x_{s}(t) d t\right| \\
& \leq\left\|\Delta A_{s}\right\|_{2}\left|\int_{0}^{1}\left\|x_{s}(t)\right\|_{2}^{2} d t\right|+2\left\|\Delta B_{s}\right\|_{2}\left|\int_{0}^{1}\left\|x_{s}(t)\right\|_{2}\left\|x_{s+1}(t)\right\|_{2} d t\right| .
\end{aligned}
$$

Substituting (15) and (16) we get

$$
\begin{aligned}
\left|\lambda_{i}-\widehat{\lambda}_{i}\right| & \leq\left\|\Delta A_{s}\right\|_{2}\left|\left(\prod_{j=0}^{\ell} \delta_{j}\right)^{2} \int_{0}^{1} d t\right|+2\left\|\Delta B_{s}\right\|_{2}\left|\prod_{j=0}^{\ell} \delta_{j} \prod_{j=1}^{\ell} \delta_{j} \int_{0}^{1} d t\right| \\
& =\left\|\Delta A_{s}\right\|_{2}\left(\prod_{j=0}^{\ell} \delta_{j}\right)^{2}+2\left\|\Delta B_{s}\right\|_{2} \delta_{0}\left(\prod_{j=1}^{\ell} \delta_{j}\right)^{2} .
\end{aligned}
$$

Remark 2. Two remarks on Theorem 4.2 are in order.

- Since the bound in (17) is proportional to the product of $\delta_{j}^{2}$, the bound can be negligibly small if $\ell$ is large and each $\delta_{j}$ is sufficiently smaller than 1 (say 0.5 ). Hence Theorem 4.2 shows that $\lambda_{i}$ is insensitive to perturbation in far-away blocks, if its separation from the spectrum of the diagonal blocks nearby the perturbed ones is large compared with the off-diagonal blocks. We illustrate this below by an example in section 5.1 .

- When the bound (13) is smaller than the Weyl bound $\|E\|_{2}$, we can obtain sharper bounds by using the results recursively, that is, the new bound (17) can be used to redefine $\delta_{j}:=$ $\frac{\left\|B_{s+j}\right\|_{2}}{\operatorname{gap}_{s+j}-\Delta-\left\|B_{s+j-1}\right\|_{2}}$, where $\Delta$ is the right-hand side of (17). The new $\delta_{j}$ is smaller than the one in (13), and this in turn yields a refined bound (17) computed from the new $\delta_{j}$.

Above we showed how a small eigenvector component implies a small eigenvalue bound. We note that Jiang [11] discusses the relation between the convergence of Ritz values obtained in the Lanczos process and eigenvector components of tridiagonal matrices. In patricular, [11] 
argues that a Ritz value must be close to an exact eigenvalue if the corresponding eigenvector of the tridiagonal submatrix has a small bottom element. We argue that Lemma 4.1 can be used to extend this to the block Lanczos method (e.g., [1, Ch. 4.6]). Assuming for simplicity that deflation does not occur, after $j$ steps of block Lanczos with block size $p$ we have $A V=V T+[0 \widehat{V} R]$ where $V \in \mathbb{C}^{n \times j p}$ and $\widehat{V} \in \mathbb{C}^{n \times p}$ have orthonormal columns and $V^{H} \widehat{V}=0 . T \in \mathbb{C}^{j p \times j p}$ is a symmetric banded matrix with bandwidth $2 p+1$, and $R \in \mathbb{C}^{p \times p}$. Then we see that letting $U=\left[V \bar{V} V_{2}\right]$ be a square unitary matrix, we have

$$
U^{H} A U=\left[\begin{array}{cccc}
T_{11} & \ddots & & \\
\ddots & \ddots & T_{j, j-1}^{H} & \\
& T_{j, j-1} & T_{j j} & R^{H} \\
& & R & A_{2}
\end{array}\right] .
$$

Note that the top-left $j p \times j p$ submatrix is equal to $T$. Now, if an eigenvalue $\lambda$ of $T$ does not belong to the last $s>0$ blocks of $T$ (which is more likely to happen if $\lambda$ is an extremal eigenvalue), then by Lemma 4.1 we can show that the bottom block $x_{p}$ of the eigenvector $x$ corresponding to $\lambda$ is small. Since the Ritz value $\lambda$ has residual $\|A y-\lambda y\|_{2} \leq\left\|x_{p}\right\|_{2}\|R\|_{2}$ where $y=U x$, this in turn implies that the there must exist an exact eigenvalue of $U^{H} A U$ lying within distance of $\|R\|_{2}\left\|x_{p}\right\|_{2}$ from the Ritz value $\lambda$.

\section{Two case studies}

Here we present two examples to demonstrate the sharpness of our approach. Specifically, we

1. Explain why aggressive early deflation can deflate many eigenvalues as "converged" when applied to the symmetric tridiagonal QR algorithm.

2. Explain why Wilkinson matrices have many pairs of nearly equal eigenvalues.

In both cases $A$ and $E$ are symmetric tridiagonal, and we denote

$$
A+E=\left[\begin{array}{cccc}
a_{1} & b_{1} & & \\
b_{1} & \ddots & \ddots & \\
& \ddots & \ddots & b_{n-1} \\
& & b_{n-1} & a_{n}
\end{array}\right],
$$

where $E$ is zero except for a few off-diagonal elements, as specified below. We assume without loss of generality that $b_{j} \geq 0$ for all $j$. Note that when $b_{j}$ are all nonzero the eigenvalues are known to be always simple [19], so the treatment of multiple eigenvalues becomes unnecessary.

In both case studies, we will bound the effect on an eigenvalue $\lambda_{i}$ of setting some $b_{j}$ to 0 . We note that Jiang [11] made the observation that setting $b_{j}$ to 0 perturbs an eigenvalue extremely insensitively if its eigenvector corresponding to the $j$ th element is negligibly small. However [11] does not explain when or why the eigenvector element tends to be negligible. Our approach throughout has been to show that for eigenvalues that are well-separated from the spectrum of the blocks nearby the perturbed blocks, the corresponding eigenvector elements can be bounded without computing them. 


\subsection{Aggressive early deflation applied to symmetric tridiagonal $Q R$}

The aggressive early deflation strategy, introduced in [4] for the nonsymmetric Hessenberg QR algorithm, is known to greatly speed up the algorithm for computing the eigenvalues of a nonsymmetric matrix by deflating converged eigenvalues long before a conventional deflation strategy does. Here we consider the simpler symmetric tridiagonal case.

We note that for symmetric tridiagonal eigenvalue problems a number of well-known algorithms exist. While the divide-and-conquer algorithm [9] is preferable when both eigenvalues and eigenvectors are required, the symmetric tridiagonal $\mathrm{QR}$ algorithm remains one of the methods of choice when only the eigenvalues are desired [7, p. 211]. Aggressive early deflation is of practical interest because it can further speed up the symmetric tridiagonal QR algorithm.

The following is a brief description of aggressive early deflation applied to the symmetric tridiagonal QR algorithm. Let $A+E$ as in (18) be a matrix obtained in the course of the algorithm. Here we let $A=\left[\begin{array}{cc}A_{1} & 0 \\ 0 & A_{2}\end{array}\right]$, where $A_{1}$ is $s \times s$ for an integer parameter $s$. $E$ has only one offdiagonal $b_{s}$. Let $A_{2}=V D V^{T}$ be an eigendecomposition, where the diagonals of $D$ are arranged in decreasing order of magnitude. Then, we have

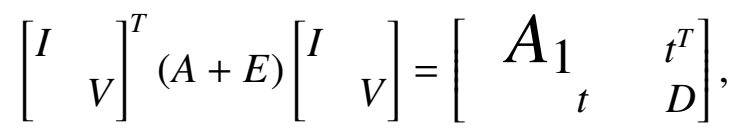

where the vector $t$ is given by $t=b_{s} V(1,:)^{T}$ where $V(1,:)$ denotes the first row of $V$. It often happens in practice that many elements of $t$ are negligibly small, in which case aggressive early deflation regards $D$ 's corresponding eigenvalues as converged and deflate them. This is the case even when none of the off-diagonals of $A$ is particularly small.

This must mean that many eigenvalues of the two matrices $A$ and $A+E$, particularly the ones that belong to the bottom-right block, must be nearly equal, or equivalently that the perturbation of the eigenvalues by the $s$ th off-diagonal $b_{s}$ is negligible. Here we give an explanation to this under an assumption that is typically valid for a tridiagonal matrix appearing in the course of the QR algorithm.

It is well-known that under mild assumptions the tridiagonal QR algorithm converges, in that the diagonals converge to the eigenvalues in descending order of magnitude, and the off-diagonal elements converge to zero [23]. In light of this, we can reasonably expect the diagonals $a_{j}$ to be roughly ordered in descending order of their magnitudes, and that the off-diagonals $b_{j}$ are small. Hence for a target (small) eigenvalue $\lambda_{i} \in \lambda\left(A_{2}\right)$, we suppose that Assumption 1 is satisfied, or that there exists an integer $\ell>0$ such that $\left|a_{j}-\lambda_{i}\right|>b_{j-1}+b_{j}+b_{s}$ for $j=1, \ldots, s+\ell$.

Under these assumptions, to bound $\left|\lambda_{i}-\widehat{\lambda}_{i}\right|$ we can use Theorem 4.2 in which we let all block sizes be 1-by-1. Since $\Delta A_{S}=0$, we have $\delta_{0}=\frac{b_{s}}{g a p_{s}-b_{s}-b_{s-1}}, \delta_{1}=\frac{b_{s+1}}{g a p_{s+1}-2 b_{s}} \delta_{j}=\frac{b_{s+j}}{g a p_{s+j}-b_{s}-b_{s+j-1}}$ for $j \geq 2$, and so using $\operatorname{gap}_{j}=\left|a_{j}-\lambda_{i}\right|$ we get

$$
\left|\lambda_{i}-\widehat{\lambda}_{i}\right| \leq 2 b_{s} \frac{b_{s}}{\left|a_{s}-\lambda_{i}\right|-b_{s}-b_{s-1}}\left(\frac{b_{s+1}}{g a p_{s+1}-2 b_{s}} \prod_{j=2}^{\ell} \frac{b_{s+j}}{\left|a_{s+j}-\lambda_{i}\right|-b_{s}-b_{s+j-1}}\right)^{2} .
$$


Simple example. To illustrate the result, let $A+E$ be the 1000-by-1000 tridiagonal matrix as in (12), where here we let $E$ be zero except for the 900th off-diagonals, which are 1 (i.e., $s=900$ ). Note that none of the off-diagonals is negligibly small. We focus on $\lambda_{i}$ (the $i$ th smallest eigenvalue of $A$ ) for $i=1, \ldots, 9$, which are smaller than 10. For such $\lambda_{i}$ we have $\ell=87$ (since $\lambda_{i} \notin$ $\left[a_{j}-\eta_{j}, a_{j}+\eta_{j}\right]=[998-j, 1004-j]$ for $j \leq 987$; recall (11)), and so (20) gives a bound

$$
\begin{aligned}
\left|\lambda_{i}-\widehat{\lambda}_{i}\right| & \leq 2 b_{s} \frac{b_{s}}{\left|a_{s}-\lambda_{i}\right|-b_{s}-b_{s-1}}\left(\frac{b_{s+1}}{g a p_{s+1}-2 b_{s}} \prod_{j=2}^{\ell} \frac{b_{s+j}}{\left|a_{s+j}-\lambda_{i}\right|-b_{s}-b_{s+j-1}}\right)^{2} \\
& =2 \frac{1}{\left|100-\lambda_{i}\right|-2}\left(\frac{1}{\left|100-1-\lambda_{i}\right|-2} \prod_{j=2}^{87} \frac{1}{\left|100-j-\lambda_{i}\right|-2}\right)^{2} \\
& <2 \frac{1}{|100-10|-2}\left(\frac{1}{|100-1-10|-2} \prod_{j=1}^{87} \frac{1}{|100-j-10|-2}\right)^{2} \\
& <5.2 \times 10^{-267}
\end{aligned}
$$

for $i=1, \ldots, 9$. This shows that all the eigenvalues of $A_{2}$ that are smaller than 10 can be hardly perturbed by setting the off-diagonal $b_{s}$ to 0 .

The same argument as above applied to $i=1, \ldots, 80$ shows that more than 80 eigenvalues of $A_{2}$ match 80 eigenvalues of $A$ to within accuracy $10^{-16}$. The general conclusion is that if the diagonal elements of $A$ are roughly graded and the off-diagonals are not too large (compared with the difference between the diagonal elements), then we can show by Theorem 4.2 that the smallest eigenvalues of $A$ are determined accurately by a much smaller lower-right submatrix of $A$.

We note that [12] shows for the non-symmetric Hessenberg QR algorithm that the process of aggressive early deflation can be regarded as extracting converged Ritz vectors by the KrylovSchur algorithm. Although we treat only the the symmetric tridiagonal case, the advantage of our analysis above is that it gives computable bounds for the accuracy of $\widehat{\lambda}_{i}$.

\subsection{Eigenvalues of Wilkinson's matrix}

Wilkinson's matrix [24] appears both in applications and for theoretical purposes, whose famous $2 n+1=21$ case is

$$
W_{21}^{+}=\left[\begin{array}{ccccccccc}
10 & 1 & & & & & & & \\
1 & 9 & \ddots & & & & & & \\
& \ddots & \ddots & 1 & & & & & \\
& & 1 & 1 & 1 & & & & \\
& & & 1 & 0 & 1 & & & \\
& & & & 1 & 1 & \ddots & & \\
& & & & & \ddots & \ddots & \ddots & \\
& & & & & & \ddots & 9 & 1 \\
& & & & & & 1 & 10
\end{array}\right] .
$$

Such matrices are known to have many pairs of extremely close eigenvalues. For example, the two largest eigenvalues of $W_{21}^{+}$agree up to about $7 \times 10^{-14}$. In general, it is observed that for 
sufficiently large $n$ the largest eigenvalues of $W_{2 n+1}^{+}$always have an extremely close pair, while smaller eigenvalues have a pair that is not as close. Wilkinson [24, p.308] notes that in general the two largest eigenvalues of the matrix $W_{2 n+1}^{+}$agree up to roughly $(n !)^{-2}$, but does not explain this in detail. We shall give an explanation using the framework we described in this paper. Define $(2 n+1)$-by- $(2 n+1)$ matrices $A$ and $E$ such that $A+E=W_{2 n+1}^{+}$by

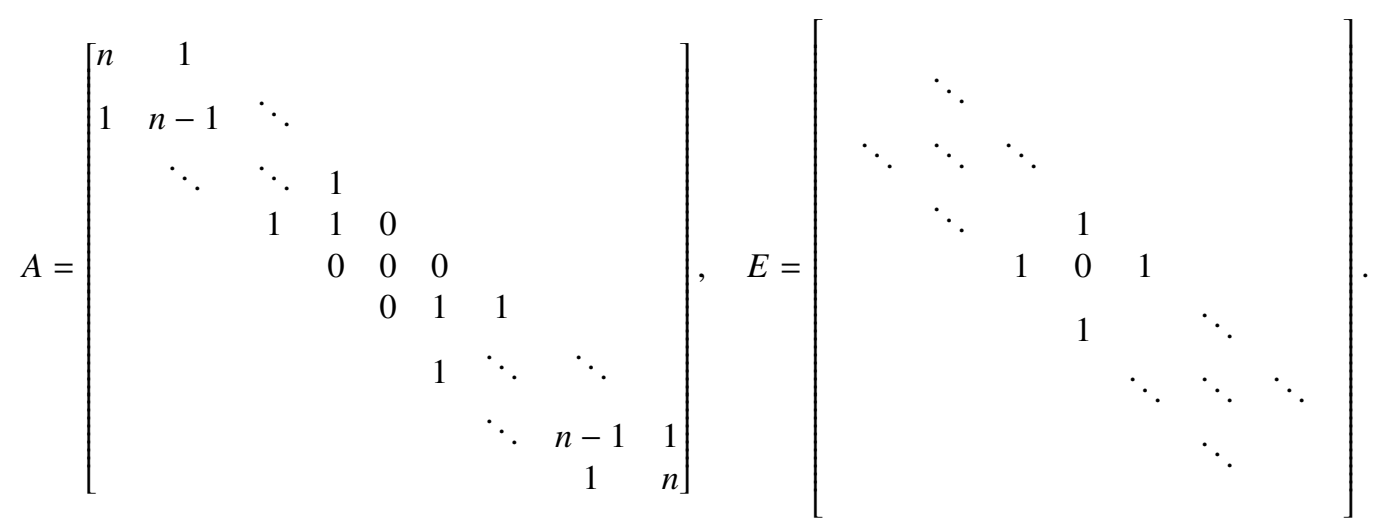

Note that $A$ has $n$ pairs of multiple eigenvalues of multiplicity 2, and a simple eigenvalue 0 . Hence to prove that large eigenvalues of $A+E=W_{2 n+1}^{+}$(those close to $n$ ) must have a very close pair, it suffices to show that large eigenvalues of $A$ are extremely insensitive to the perturbation $E$.

\subsubsection{Bounding $\left|\widehat{\lambda}_{i}-\lambda_{i}\right|$}

Unfortunately, the setting (23) does not satisfy Assumption 1 that is required in Theorem 4.2, because any large eigenvalue of $A$ belongs to some of the top and bottom blocks, which here are simply the diagonal elements. For example, the two largest eigenvalues of $A$ belong to the top and bottom blocks.

Here we show that by a slight modification of the derivation of Theorem 4.2 we can still get sharp bounds for $\left|\lambda_{i}-\widehat{\lambda}_{i}\right|$ for large $i$.

Below we suppose $n>4$. First we consider the two largest eigenvalues of $A$, either of which we denote by $\lambda_{i}$ (i.e., $i$ can be either $2 n$ or $2 n+1$ ). As before, define $x(t)=\left[x_{1}(t) \ldots x_{2 n+1}(t)\right]^{H}$ such that $(A+t E) x(t)=\lambda_{i}(t) x(t)$.

First, the $(n+1)$ th row of $(A+t E) x(t)=\lambda_{i}(t) x(t)$ is

$$
\lambda_{i}(t) x_{n+1}(t)=t\left(x_{n}(t)+x_{n+2}(t)\right),
$$

hence

$$
\left|x_{n+1}(t)\right| \leq \frac{2 t \max \left(\left|x_{n}(t)\right|,\left|x_{n+2}(t)\right|\right)}{\left|\lambda_{i}(t)\right|}, \quad t \in[0,1] .
$$

We separately consider the two cases $\left|x_{n}(t)\right| \geq\left|x_{n+2}(t)\right|$ and $\left|x_{n}(t)\right|<\left|x_{n+2}(t)\right|$, and show that in both cases a tight bound can be obtained for $\left|\lambda_{i}(A+E)-\lambda_{i}(A)\right|$.

Suppose $\left|x_{n}(t)\right| \geq\left|x_{n+2}(t)\right|$. Then we also have $\left|x_{n}(t)\right| \geq\left|x_{n+1}(t)\right|$ in view of (24). From the $n$th row of $(A+t E) x(t)=\lambda_{i}(t) x(t)$ we similarly get

$$
\left|x_{n}(t)\right| \leq \frac{\left|x_{n-1}(t)\right|+\left|x_{n+1}(t)\right|}{\left|\lambda_{i}(t)-1\right|}, \quad t \in[0,1] .
$$


Now since $n<\lambda_{i}(t)<n+1$ for all $t \in[0,1]^{3}$ we must have $\left|x_{n-1}(t)\right| \geq\left|x_{n}(t)\right| \geq\left|x_{n+1}(t)\right|$. Substituting this into (25) yields $\left|x_{n}(t)\right| \leq \frac{\left|x_{n-1}(t)\right|}{\left|\lambda_{i}(t)-1\right|-1}$. Therefore we have

$$
\left|x_{n}(t)\right| \leq \frac{\left|x_{n-1}(t)\right|}{n-2} \quad \text { for } \quad t \in[0,1]
$$

By a similar argument we find for all $t \in[0,1]$ that

$$
\left|x_{n-j}(t)\right| \leq \frac{\left|x_{n-j-1}(t)\right|}{n-j-2} \quad \text { for } \quad j=0, \ldots, n-3 .
$$

Hence together with (24) we get

$$
\begin{aligned}
\left|x_{n+1}(t)\right| & \leq \frac{2 t}{n-1}\left|x_{2}(t)\right| \prod_{j=0}^{n-3} \frac{1}{n-j-2} \leq \frac{2 t}{n-1} \prod_{j=0}^{n-3} \frac{1}{n-j-2}, \\
\left|x_{n}(t)\right| & \leq \prod_{j=0}^{n-3} \frac{1}{n-j-2} .
\end{aligned}
$$

We now plug these into (4) to get

$$
\begin{aligned}
\left|\lambda_{i}(A+E)-\lambda_{i}(A)\right| & \leq\left|\int_{0}^{1} x(t)^{H} \operatorname{Ex}(t) d t\right| \\
& \leq \int_{0}^{1} 2\left(\left|x_{n}(t)\right|+\left|x_{n+2}(t)\right|\right)\left|x_{n+1}(t)\right| d t \\
& \leq \frac{4}{n-1}\left(\prod_{j=0}^{n-3} \frac{1}{n-j-2}\right)^{2} \int_{0}^{1} t d t \\
& =\frac{2}{n-1}\left(\prod_{j=0}^{n-3} \frac{1}{n-j-2}\right)^{2} .
\end{aligned}
$$

The case $\left|x_{n}(t)\right|<\left|x_{n+2}(t)\right|$ can also be treated similarly, and we get the same result.

Finally, since (29) holds for both $i=2 n$ and $i=2 n+1$, we conclude that

$$
\left|\lambda_{2 j}\left(W_{2 n+1}^{+}\right)-\lambda_{2 j+1}\left(W_{2 n+1}^{+}\right)\right| \leq \frac{4}{n-1}\left(\prod_{j=0}^{n-3} \frac{1}{n-i-2}\right)^{2} .
$$

We easily appreciate that the bound (30) roughly scales as $1 /(n-1)((n-2) !)^{2}$ as $n \rightarrow \infty$, which supports the claim in [24].

\footnotetext{
${ }^{3}$ We can get $n<\lambda(t)<n+1$ by first following the same argument using $n-\|E\|_{2}<\lambda(t)<n+1$, which follows from Weyl's and Gerschgorin's theorems.
} 
We also note that by a similar argument we can prove for $j \geq 1$ that the $2 j-1$ th and $2 j$ th largest eigenvalues of $W_{2 n+1}^{+}$match to $O\left((n-j)^{-1}((n-j-1) !)^{-2}\right)$, which is small for small $j$, but not as small for larger $j$. This is an accurate description of what is well known about the eigenvalues of Wilkinson matrices.

In [25] Ye investigates tridiagonal matrices with nearly multiple eigenvalues, motivated also by the Wilkinson matrix. We note that we can give another explanation for the nearly multiple eigenvalue by combining ours with Ye's. Specifically, we first consider the block partition $W_{2 n+1}^{+}=$ $\left[\begin{array}{cc}W_{1} & E^{H} \\ E & W_{2}\end{array}\right]$ where $W_{1}$ is $(n+1)$-by- $(n+1)$, and $E$ contains one off-diagonal of $W_{2 n+1}^{+}$. We can use Theorem 4.2 to show that the largest eigenvalues of $W_{1}$ and $W_{2}$ are nearly the same; let the distance be $\delta$. Furthermore, we can use Lemma 4.1 to show the corresponding eigenvectors decay exponentially, so that the eigenvector component for $W_{1}$ is of order $1 / n$ ! at the bottom, and that for $W_{2}$ is of order $1 / n$ ! at the top. We can then use Theorem 2.1 of [25] to show that $W_{2 n+1}^{+}$must have two eigenvalue within distance $\delta+O(1 / n !)$. However, this bound is not as tight as the bound (30), being roughly its square root.

\section{Appendix A. Multiple eigenvalues}

In the text we assumed that all the eigenvalues of $A+t E$ are simple for all $t \in[0,1]$. Here we treat the case where multiple eigenvalues exist, and show that all the results we proved still hold exactly the same.

We note that $[2,3]$ indicate that multiple eigenvalues can be ignored when we take integrals such as (3), because $A+t E$ can only have multiple eigenvalues on a set of $t$ of measure zero, and hence (1) can be integrated on $t$ such that $A+t E$ has only simple eigenvalues. However when $A, E$ are both allowed to be arbitrary Hermitian matrices ${ }^{4}$ we cannot use this argument, which can be seen by a simple counterexample $A=E=I$, for which $A+t E$ has a multiple eigenvalue for all $0 \leq t \leq 1$. Hence in a general setting we need a different approach.

\section{Appendix A.1. Multiple eigenvalue first order perturbation expansion}

First we review a known result on multiple eigenvalue first order perturbation expansion [22, 16, 13]. Suppose that a Hermitian matrix $A$ has a multiple eigenvalue $\lambda_{0}$ of multiplicity $r$. There exists a unitary matrix $Q=\left[Q_{1}, Q_{2}\right]$, where $Q_{1}$ has $r$ columns, such that

$$
Q^{H} A Q=\left[\begin{array}{cc}
\lambda_{0} I_{r} & 0 \\
0 & \Lambda
\end{array}\right]
$$

where $\Lambda$ is a diagonal matrix that contains eigenvalues not equal to $\lambda_{0}$. Then, the matrix $A+\varepsilon E$ has eigenvalues $\widehat{\lambda}_{1}, \widehat{\lambda}_{2}, \ldots, \widehat{\lambda}_{r}$ admitting the first order expansion

$$
\widehat{\lambda}_{i}=\lambda_{0}+\mu_{i}\left(Q_{1}^{H} E Q_{1}\right) \varepsilon+o(\varepsilon), \quad \text { for } \quad i=1, \ldots, r,
$$

where $\mu_{i}\left(Q_{1}^{H} E Q_{1}\right)$ denotes the $i$ th eigenvalue of the $r$-by-r matrix $Q_{1}^{H} E Q_{1}$.

\footnotetext{
${ }^{4}$ The argument in [3] assumes that $\lambda$ is a simple eigenvalue at $t=0$.
} 
Using (A.2), we obtain the partial derivative corresponding to (1) when $A+t E$ has a multiple eigenvalue $\lambda_{i}(t)=\lambda_{i+1}(t)=\cdots=\lambda_{i+r-1}(t)$ of multiplicity $r$, with corresponding invariant subspace $Q_{1}(t)$ :

$$
\frac{\partial \lambda_{i+j-1}(t)}{\partial t}=\mu_{j}\left(Q_{1}(t)^{H} E Q_{1}(t)\right) \quad \text { for } \quad j=1, \ldots, r .
$$

Now, let $Q_{1}(t)^{H} E Q_{1}(t)=U^{H} D U$ be the eigendecomposition in which the diagonals of $D$ are arranged in descending order. Then $D=U Q_{1}(t) E Q_{1}(t) U^{H}=\tilde{Q}_{1}(t) E \tilde{Q}_{1}(t)$, where $\tilde{Q}_{1}(t)=Q_{1}(t) U^{H}$, so $\mu_{j}\left(Q_{1}(t)^{H} E Q_{1}(t)\right)=q_{j}(t)^{H} E q_{j}(t)$, where $q_{j}(t)$ denotes the $j$ th column of $\tilde{Q}_{1}(t)$. Therefore we can write

$$
\frac{\partial \lambda_{i+j-1}(t)}{\partial t}=q_{j}(t)^{H} E q_{j}(t) \text { for } j=1, \ldots, r .
$$

Now, since any vector of the form $Q_{1}(t) v$ is an eigenvector corresponding to the eigenvalue $\lambda_{i}(t)$, so is $q_{j}(t)$. We conclude that we can always write the first order perturbation expansion of $\lambda_{i}(t)$ in the form (1), in which when $\lambda_{i}(t)$ is a multiple eigenvalue $x(t)$ represents a particular eigenvector among the many possible choices.

Finally, since all our eigenvector bounds (such as (5), (15) and (16)) hold regardless of whether $\lambda_{i}$ is a multiple eigenvalue or not, we conclude that all the bounds in the text hold exactly the same without the assumption that $\lambda_{i}(t)$ is simple for all $t \in[0,1]$.

\section{Appendix A.2. Note on the trailing term}

Here we refine the expansion (A.2) by showing that the trailing term is $O\left(\varepsilon^{2}\right)$ instead of $o(\varepsilon)$. To show this, we recall (A.1) and see that

$$
Q^{H}(A+\varepsilon E) Q=\left[\begin{array}{cc}
\lambda_{0} I_{r}+\varepsilon Q_{1}^{H} E Q_{1} & \varepsilon Q_{1}^{H} E Q_{2} \\
\varepsilon Q_{2}^{H} E^{H} Q_{1} & \Lambda+\varepsilon Q_{2}^{H} E Q_{2}
\end{array}\right]
$$

For sufficiently small $\varepsilon$ there is a positive gap in the spectrums of the matrices $\lambda_{0} I_{r}+\varepsilon Q_{1}^{H} E_{11} Q_{1}$ and $\Lambda+\varepsilon Q_{2}^{H} E_{22} Q_{2}$. Hence, using the quadratic eigenvalue perturbation bounds in [14] and the fact $\left\|\varepsilon Q_{1}^{H} E Q_{2}\right\|_{2} \leq\|\varepsilon E\|_{2}$ we see that the $i$ th eigenvalue of $A+\varepsilon E$ and those of the matrix $\left[\begin{array}{cc}\lambda_{0} I_{r}+\varepsilon Q_{1}^{H} E Q_{1} & 0 \\ 0 & \Lambda+\varepsilon Q_{2}^{H} E Q_{2}\end{array}\right]$ differ at most by $\frac{2\|\varepsilon E\|_{2}^{2}}{g a p+\sqrt{g a p^{2}+4\|\varepsilon E\|_{2}^{2}}}$. This is of size $O\left(\varepsilon^{2}\right)$ because gap $>0$. Therefore we conclude that (A.2) can be replaced by

$$
\widehat{\lambda}_{i}=\lambda_{0}+\mu_{i}\left(Q_{1}^{H} E Q_{1}\right) \varepsilon+O\left(\varepsilon^{2}\right) \text { for } i=1,2, \ldots, r .
$$

Acknowledgment. I am thankful to the referee for providing helpful suggestions, particularly for pointing out many connections with related work in the literature.

\section{References}

[1] Z. Bai, J. Demmel, J. Dongarra, A. Ruhe, H. van der Vorst, Templates for the Solution of Algebraic Eigenvalue Problems: A Practical Guide, SIAM, Philadelphia, USA, 2000.

[2] J. Barlow, J. Demmel, Computing accurate eigensystems of scaled diagonally dominant matrices, SIAM J. Numer. Anal. 27 (3) (1990) 762-791. 
[3] J. Barlow, I. Slapnicar, Optimal perturbation bounds for the Hermitian eigenvalue problem, Linear Algebra Appl. 309 (1-3) (2000) 19-43.

[4] K. Braman, R. Byers, R. Mathias, The Multishift QR Algorithm. PART II: Aggressive Early Deflation, SIAM J. Matrix Anal. Appl. 23 (2002) 948-973.

[5] J. J. M. Cuppen, A divide and conquer method for the symmetric tridiagonal eigenproblem., Numer. Math. 36 (1981) 177-195.

[6] C. Davis, W. M. Kahan, The rotation of eigenvectors by a perturbation. III, SIAM J. Numer. Anal. 7 (1) (1970) $1-46$.

[7] J. Demmel, Applied Numerical Linear Algebra, SIAM, Philadelphia, USA, 1997.

[8] G. H. Golub, C. F. Van Loan, Matrix Computations, The Johns Hopkins University Press, 1996.

[9] M. Gu, S. C. Eisenstat, A divide-and-conquer algorithm for the symmetrical tridiagonal eigenproblem, SIAM J. Matrix Anal. Appl. 16 (1) (1995) 172-191.

[10] I. C. F. Ipsen, B. Nadler, Refined perturbation bounds for eigenvalues of Hermitian and non-Hermitian matrices, SIAM J. Matrix Anal. Appl. 31 (1) (2009) 40-53.

[11] E.-X. Jiang, Perturbation in eigenvalues of a symmetric tridiagonal matrix, Linear Algebra Appl. 399 (Sp. Iss. SI) (2005) 91-107.

[12] D. Kressner, The effect of aggressive early deflation on the convergence of the QR algorithm, SIAM J. Matrix Anal. Appl. 30 (2) (2008) 805-821.

[13] D. Kressner, M. Jose Pelaez, J. Moro, Structured Hölder condition numbers for multiple eigenvalues, SIAM J. Matrix Anal. Appl. 31 (1) (2009) 175-201.

[14] C.-K. Li, R.-C. Li, A note on eigenvalues of perturbed Hermitian matrices, Linear Algebra Appl. 395 (2005) 183-190.

[15] R. Mathias, Quadratic residual bounds for the Hermitian eigenvalue problem, SIAM J. Matrix Anal. Appl. 19 (2) (1998) 541-550.

[16] J. Moro, J. V. Burke, M. L. Overton, On the Lidskii-Vishik-Lyusternik perturbation theory for eigenvalues of matrices with arbitrary Jordan structure, SIAM J. Matrix Anal. Appl. 18 (4) (1997) 793-817.

[17] B. Parlett, C. Vömel, Detecting localization in an invariant subspace. In preparation.

[18] B. N. Parlett, Invariant subspaces for tightly clustered eigenvalues of tridiagonals, BIT Numerical Mathematics 36 (3) (1996) 542-562.

[19] B. N. Parlett, The Symmetric Eigenvalue Problem, SIAM, Philadelphia, 1998.

[20] B. N. Parlett, A result complementary to Gersgorin's circle theorem, Linear Algebra Appl. 431 (1-2) (2009) 20-27.

[21] G. W. Stewart, J.-G. Sun, Matrix Perturbation Theory, Academic Press, 1990.

[22] J.-G. Sun, On condition numbers of a nondefective multiple eigenvalue, Numer. Math. 61 (2) (1992) $265-275$.

[23] L. N. Trefethen, D. Bau, Numerical Linear Algebra, SIAM, Philadelphia, USA, 1997.

[24] J. H. Wilkinson, The Algebraic Eigenvalue Problem, Oxford University Press, USA, 1965.

[25] Q. Ye, On close eigenvalues of tridiagonal matrices, Numer. Math. 70 (1995) 507-514. 